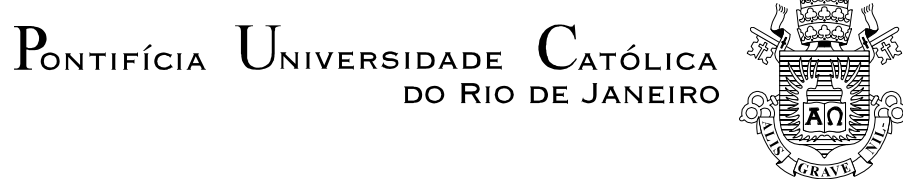

Georges Eduardo Gerbauld Catalão

\title{
A Relação de "Lead-lag" entre o Índice Dow Jones Industrial Average futuro e à vista
}

Dissertação de Mestrado

Dissertação apresentada como requisito parcial para obtenção do grau de Mestre pelo Programa de Pós-graduação em Administração de Empresas do Departamento de Administração da PUC-Rio.

Orientador: Marcelo Cabús Klotzle 


$$
\begin{array}{r}
\text { Pontifícia Universidade C Católica } \\
\text { Do RIo de JANEIRo }
\end{array}
$$

Georges Eduardo Gerbauld Catalão

\begin{abstract}
A Relação de Lead-lag entre o Índice Dow Jones Industrial Average futuro e à vista
\end{abstract}

Dissertação apresentada como requisito parcial para obtenção do grau de Mestre pelo Programa de Pós-graduação em Administração de Empresas do Departamento de Administração da PUC-Rio.

\author{
Prof. Marcelo Cabús Klotzle \\ Orientador \\ IAG - PUC-Rio
}

Prof. Walter Lee Ness

IAG - PUC-Rio

Prof. Katia Rocha

IPEA RJ

Prof. Nizar Messari

Coordenador Setorial do Centro

Técnico Científico - PUC-Rio

Rio de Janeiro, 3 de fevereiro de 2009 
Todos os direitos reservados. É proibida a reprodução total ou parcial do trabalho sem autorização da universidade, do autor e do orientador.

\section{Georges Eduardo Gerbauld Catalão}

Graduou-se em Ciências Econômicas no IBMEC-RJ (Instituto Brasileiro de Mercado de Capitais do Rio de Janeiro) em 2004. Trabalhou no BackOffice do Banco BBM de 2003 a 2005. Trabalhou na IBM Brazil na área de Incentivos e Comissões de 2005 a 2008. Trabalha na Triscorp Invstimentos na área de Fundo de Fundos e Client Advisory desde 2008.

Ficha Catalográfica

Catalão, Georges Eduardo Gerbauld

A relação de "Lead-lag" entre o índice Dow Jones Industrial Average futuro e à vista / Georges Eduardo Gerbauld Catalão ; orientador: Marcelo Cabús Klotzle $-2009$.

51 f. : il. ; $30 \mathrm{~cm}$

Dissertação (Mestrado em Administração)Pontifícia Universidade Católica do Rio de Janeiro, Rio de Janeiro, 2009.

Inclui bibliografia

1. Administração - Teses. 2. Efeito "Lead-lag". 3. Ineficiência de mercado. 4. Índice Dow Jones Industrial Average. 5. Mercado futuro. 6. Mercado à vista. 7. Teste de Dickey-Fuller aumentado. 8. Regressão por mínimos quadrados ordinários. 9. Regressão por mínimos quadrados em dois estágios. 10. Modelo vetorial de correção de erro. I. Klotzle, Marcelo Cabús. II. Pontifícia Universidade Católica do Rio de Janeiro. Departamento de Administração. III. Título. 


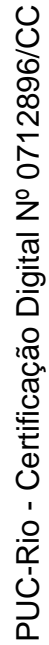

Para minha mulher, Carolina, pelo apoio e incentivo. 


\section{Agradecimentos}

Ao meu orientador Professor Marcelo Cabús Klotzle pelo estímulo e parceria para a realização deste trabalho.

A PUC-Rio, pelos auxílios concedidos, sem os quais este trabalho não poderia ter sido realizado.

A minha mulher que por tantas vezes me apoio e estimulou no decorrer do mestrado.

A minha família que sempre me incentivo e esteve presente até mesmo na minha defesa de dissertação.

A minha mãe e meu pai que tornaram possível que chegasse onde estou.

Aos professores que participaram da Comissão examinadora. 


\section{Resumo}

Catalão, Georges Eduardo Gerbauld; Klotzle, Marcelo Cabús. A Relação de “Lead-lag” entre o Índice Dow Jones Industrial Average futuro e à vista. Rio de Janeiro, 2009. 51p. Dissertação de Mestrado - Departamento de Administração, Pontifícia Universidade Católica do Rio de Janeiro.

Como o advento da internet os mercados estão cada vez mais integrados e arbitrados. A possibilidade de se comprar e vender ativos instantaneamente através da internet contribuiu para a eficiência de mercado, ou seja, ficou mais fácil difundir informações novas e para se reagir em cima dessas informações. A teoria de mercados eficientes estabelece que os preços dos ativos devam responder instantaneamente a novas informações, logo, não havendo espaço para arbitragem. Todavia, muitas vezes isso não é verificado no mundo real. Ao contrário do que a teoria de mercados eficientes estabelece, existem fatores que tornam os mercados assimétricos e ineficientes. Um exemplo de fonte geradora de ineficiência é o custo de transação que muitas vezes impossibilita a prática de arbitragem. Outra questão fundamental geradora de assimetria entre mercados à vista e mercados futuros é a possibilidade de se operar muito mais alavancado no mercado futuro do que no à vista, pois só é necessário disponibilizar uma fração do montante utilizado na operação. Esses fatores unidos ocasionam um efeito de mercado conhecido no meio acadêmico como efeito "lead-lag". Isto é, existe um ativo que antecipa (lead) o movimento do outro ativo (lag), em outras palavras, podemos dizer que um ativo reage primeiro a novas informações que o outro.

\section{Palavras-chave}

Efeito "Lead-lag"; ineficiência de mercado; assimetria de informação; índice Dow Jones Industrial Average; mercado futuro; mercado à vista; teste de DickeyFuller Aumentado; regressão por Mínimos Quadrados Ordinários. 


\section{Abstract}

Catalão, Georges Eduardo Gerbauld; Klotzle, Marcelo Cabús. The Lead-lag relatioship between the spot and future Dow Jones Industrial Average Index. Rio de Janeiro, 2009. 51p. MSc Dissertation - Departamento de Administração, Pontifícia Universidade Católica do Rio de Janeiro.

With the internet birth, markets are more connected and accurately priced each day. The possibility to buy and sell assets online contributed to markets efficiency, hence now it's easier to spread and price new information. The efficient market theory says that assets prices must react instantly to new information, thus there should be no space for arbitrage. Nevertheless this is frequently not true in the real world. In contrast with what the efficient market theory establishes, there are several factors that contribute to market inefficiency and asymmetry. A source of market inefficiency, for instance, is the transactional cost, which many times turn arbitrages practice impossible. Another example that contributes to market asymmetry is the possibility to leverage much more in the future markets than in the spot markets, since just a small fraction of the traded amount is required. These issues putted together create an effect known in the academy as the Lead-lag effect. More precisely, there is an asset that anticipates (lead) the movement of another asset (lag), in other words, we can say that one asset reacts faster to new information than the other one.

\section{Keywords}

Lead-lag Effect; market inefficiency; information asymmetry; Dow Jones Industrial Average Index; future market; spot market; Augmented Dickey-Fuller test; Ordinary Least Square regression. 


\section{Sumário}

1. Introdução 12

1.1. Formulação do Problema 12

1.2. Objetivos 13

1.3. Justificativa e Relevância 13

1.4. Delimitação do Estudo 14

2. Referencial Teórico 15

3. Dados 21

3.1. História 21

3.2. Especificações Contratuais 22

3.3. Especificações Amostrais 22

4. Modelagem 24

4.1. Estacionaridade 24

4.2. Teste de Dickey-Fuller 25

4.3. Teste de Duas Etapas de Engle-Granger 26

4.4. Teste de Causalidade de Granger 27

4.5. Metodologia dos Mínimos Quadrados em Dois Estágios 28

4.6. Teste de Co-integração de Johansen e Juselius 28

4.7. Modelo Vetorial de Correção de Erro 29

4.8. Teste de Heterocedasticidade de White 30

4.9. Modelo GARCH 30

5. Resultados 32

5.1. Séries Log-preço 32

5.2. Séries Log-retorno 33

5.3. Co-integração por Engle-Granger 33 
5.4. Testando a Teoria Inversa 35

5.5. Teste de Causalidade de Granger 37

5.6. Refazendo a Regressão por TSLS 38

5.7. Co-integração por Johansen 39

5.8. Análise Intertemporal (VECM) 41

5.9. Teste de Auto-correlação 44

5.10. Modelo GARCH 45

6. Conclusão 48

7. Referências Bibliográficas 50 


\section{Lista de Figuras}

Figura 1 - Exemplo de séries não estacionárias. Dow Jones

Industrial Average à vista e futuro

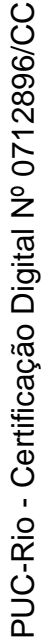




\section{Lista de Tabelas}

Tabela 1 - Teste de Dickey-Fuller para série log-preço futuro 32

Tabela 2 - Teste de Dickey-Fuller para série log-preço à vista 32

Tabela 3 - Teste de Dickey-Fuller para série log-retorno futuro 33

Tabela 4 - Teste de Dickey-Fuller para série log-retorno à vista 33

Tabela 5 - Regressão por OLS com log-preço à vista sendo a variável dependente 34

Tabela 6 - Teste de Dickey-Fuller para a regressão com log-preço à vista sendo a variável dependente $\quad 35$

Tabela 7 - Regressão por OLS com log-preço futuro sendo a variável dependente $\quad 36$

Tabela 8 - Teste de Dickey-Fuller para a regressão com log-preço futuro sendo a variável dependente 36 Tabela 9 - Teste de Causalidade de Granger 37

Tabela 10 - Regressão por Mínimos Quadrados em Dois Estágios 38 Tabela 11 - Teste de Dickey-Fuller para o resíduo da regressão por TSLS 39

Tabela 12 - Teste de Co-integração de Johansen 40

Tabela 13 - Teste Vetorial de Correção de Erro 42

Tabela 14 - Teste de Heterocedasticidade de White para a regressão por OLS 44

Tabela 15 - Teste de Heterocedasticidade de White para a regressão por TSLS 45

Tabela 16 - Teste GARCH 46 